\title{
Prolonged QT Interval, Coronary Artery Fistula and Ventricular Fibrillation during Diagnostic Coronary Angiography, A Conglomeration of Three Rare Entities in a Patient: Case Report and Literature Review
}

\author{
Imran Hameed* \\ Consultant Cardiologist, Ziauddin Medical University, Pakistan
}

Submission: March 31, 2020; Published: April 08, 2020

*Corresponding author: Imran Hameed, Consultant Cardiologist, Ziauddin Medical University, Ankle Saria Nursing Home, Sir Aga Khan III Road, Karachi, Pakistan

Abstract

Case of a 55 years old lady is reported who underwent diagnostic coronary angiography and had prolonged QT interval on resting ECG. Coronary angiogram showed no significant stenosis, however, a small Coronary Cameral Fistula was noted originating from proximal Right Coronary artery and draining into Right Atrium. After the last injection into RCA she developed Torsades de pointes which degenerated into Ventricular Fibrillation. Promptly defibrillated, the patient remained asymptomatic and stable. Constellation of three rare entities, i.e. Prolonged QT interval, Coronary artery fistula and patient developing Ventricular fibrillation during coronary angiography constitute a rare combination. The case report and a brief literature review of all the three entities are incorporated in this manuscript.

Keywords: Coronary angiography; Coronary artery fistula; Ventricular fibrillation; Prolonged QT interval

Abbreviations: ECG: Echocardiography; RCA: Right Coronary Artery; LCx: Left Circumflex; LAD: Left Anterior Descending; CAF: Coronary Artery Fistula; VF: Ventricular Fibrillation; CCS: Canadian Cardiovascular Society; HEENT: Head, Eyes Ear, Nose and Throat

\section{Introduction}

QT interval is the total time of ventricular depolarization and repolarization and, on surface ECG, is measured from the onset of $Q$ wave till the termination of $T$ wave [1]. A prolongation of it due to either congenital or acquired reasons predisposes to ventricular arrhythmias, the most sinister being Torsades de Pointes and Ventricular Fibrillation. It is a disease of "ion channels" responsible for myocyte action potential either by gain or loss of function.

Coronary angiography came into clinical practice in the early 60 s. Since then it has taken rapid strides and from the realms of diagnostic procedures has now become an integral part of the therapeutic armamentarium of cardiology. With newer techniques, thousands of such procedures are done on a daily basis all over the world making it a safe procedure but some complications are still being encountered. Among the arrhythmic complications, Ventricular fibrillation is the most dreaded one and if not dealt with effectively the consequences could be none less than fatal.

Coronary artery fistula is an abnormal communication between a coronary artery or its branch with either a cardiac chamber or with another blood vessel directly (without the intervening capillary bed) [2]. This entity is very rare and usually diagnosed during coronary angiography as an incidental finding. Management depends upon the size of fistula and symptoms produced by it.

\section{Case Presentation}

A 55 years old female, diabetic (type II) and hypertensive, presented with chest pain (CCS Angina grade III). Physical examination showed weight of $75 \mathrm{KGs}$ and a height of $170 \mathrm{cms}$ giving BMI of $26 \mathrm{~kg} / \mathrm{m}^{2}$ (overweight) and BSA of $1.88 \mathrm{~m}^{2}$. Her vitals were normal with a heart rate of $80 \mathrm{bpm}$ and BP of $120 / 75 \mathrm{mmHg}$. No significant findings were noted on HEENT examination. 


\section{Juniper Online Journal of Case Studies}

Her chest was clear on auscultation whereas CVS examination showed all pulses normal with S4 (added sound) heard at apex. Neurological examination showed no cognitive, attention or sensori-motor deficit.

Her hematological and biochemical profile were within normal limits. A standard 12 lead surface ECG showed normal Sinus Rhythm with evidence of Left Atrial enlargement and Left
Ventricular Hypertrophy (Cornell index $=28 \mathrm{~mm}$ ). QTc interval was $492 \mathrm{msec}$ in lead V5 (Figure 1), by Linear regression formula [3]. Transthoracic echo revealed mild left ventricular (eccentric) hypertrophy $\left(\mathrm{LVMI}=126.5 \mathrm{G} / \mathrm{m}^{2} ; \mathrm{RWT}=0.32\right)$ with an Ejection Fraction of $60 \%$ (modified Bi-plane Simpson's method). No wall motion abnormality was seen. Diastolic dysfunction, grade I was noted, with no significant valvular pathology.

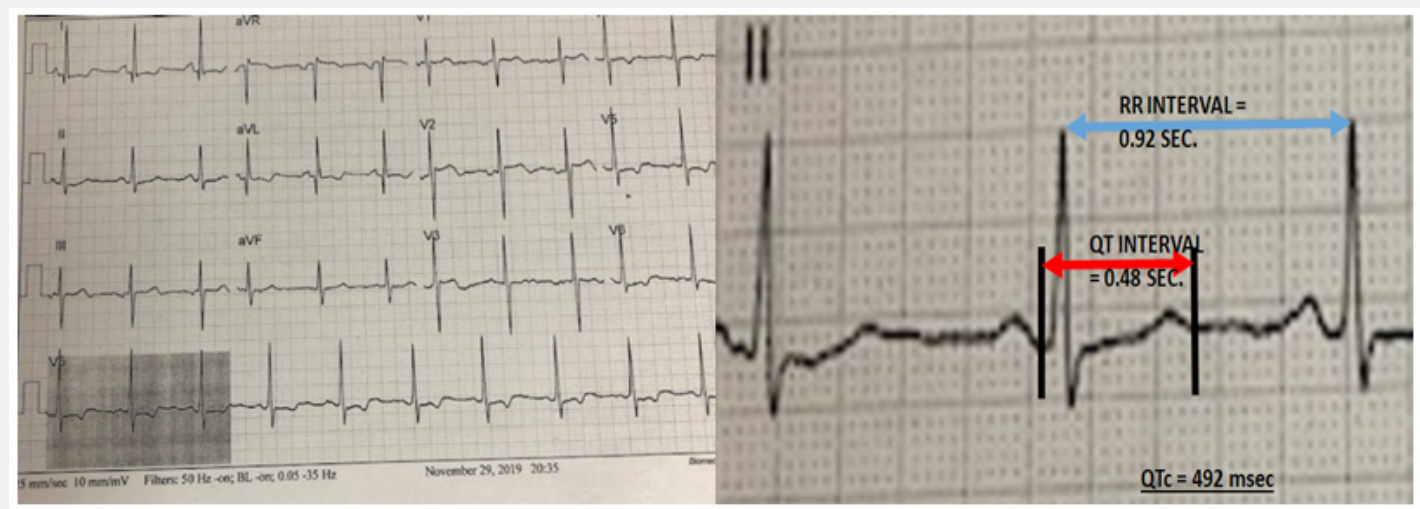

Figure 1: 12 Lead surface ECG and QTc interval.

She had a Myocardial perfusion scan (SPECT study) done about two months prior, which showed mild reversible ischemia of apical region after an exercise of 6 minutes. She was on a combination of Regular and NPH insulin for her diabetes, and also on beta blockers and angiotensin converting enzyme inhibitor. Statin, antiplatelet, nitrates and Trimatazidine were added with beta blocker dose intensified. She reported no significant improvement in her symptoms two weeks later and based on her risk factors, symptoms and findings of Perfusion study, Coronary Angiography was planned which the patient also insisted upon.

\section{Coronary angiography}

Through Right Femoral artery by Seldinger's technique,
Coronary angiography was done by selective Judkin's catheters 6F. Non-ionic, low osmolality (Ultravist 370 ) was used (55cc). No significant stenosis or ectasia was noted, however, there was increased tortuosity of vessels likely due to left ventricular hypertrophy. A small Coronary cameral fistula, Figure 2, was seen arising from proximal right coronary artery and draining into Right Atrium. After last injection to Right Coronary Artery near the end of Cine recording, the patient suddenly went into Torsades de Pointes which immediately degenerated into Ventricular fibrillation. The beginning of this arrhythmia can be noted in the image of RCA angiogram (Figure 2B). The patient remained totally asymptomatic, fully conscious and responsive. Sinus Rhythm was re-established by a single DC shock of 200 Joules.

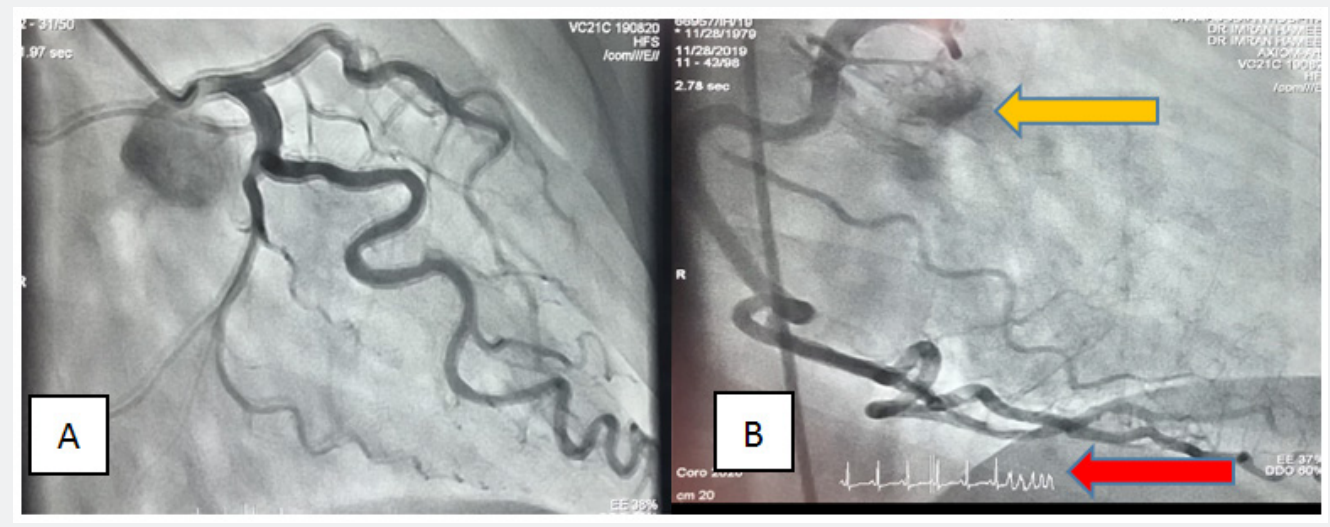

Figure 2: Coronary Angiogram: A-Left, B- Right, yellow arrow shows coronary cameral fistula from proximal RCA to right atrium, red arrow shows initiation of ventricular arrhythmia. 


\section{Juniper Online Journal of Case Studies}

\section{Discussion}

There is no literature report of three entities occurring simultaneously, i.e. prolonged QT interval, coronary artery fistula and patient going into Ventricular fibrillation during diagnostic coronary angiography, which is perhaps a first. Literature is ripe with such events occurring individually and a discussion of these entities is put henceforth.

\section{Coronary artery fistula}

Coronary artery fistula is an abnormal communication between a Coronary artery and either a cardiac chamber (coronary cameral) or systemic/pulmonary vasculature (could be another coronary artery, pulmonary artery, cardiac veins, superior vena cava or bronchial artery) without an intervening capillary network.

Mainly there are two types of cardiac fistulae, congenital and acquired, the former being more common. The causes of acquired fistulae are multiple.
Prevalence of fistulae can be gauged from findings of large studies of coronary angiography only, as these entities are recognized incidentally. Incidence of Coronary Artery Fistula in congenital heart disease has been reported as $0.2-0.4 \%$. However, in general population it is $0.002 \%$. [4]. Manolis Vavuranakis et al. [5] in their study of 33,600 diagnostic cardiac catheterization, found $34(0.1 \%)$ cases with CAF [5].

According to a large study by Levin, the frequency of origin of these fistulae (artery of origin), in descending order, are: Right coronary artery (50\%), Left coronary artery (42\%) and rest from both (multiple) [6]. Pathophysiologically, the resistance of fistulous connections and the site of drainage are very important. The site of drainage according to decreasing prevalence in another study were found to be: Right ventricle (41\%), Right atrium (26\%), Pulmonary artery (15-30\%), Coronary sinus and cardiac veins $(7 \%)$ Left atrium and Left ventricle (3-5\%), and Bronchial arteries $(0.6 \%)$ [7]. The same study has given an exhaustive classification of these fistulae, Table 1.

Table 1: Classification of coronary artery fistula based on various parameters.

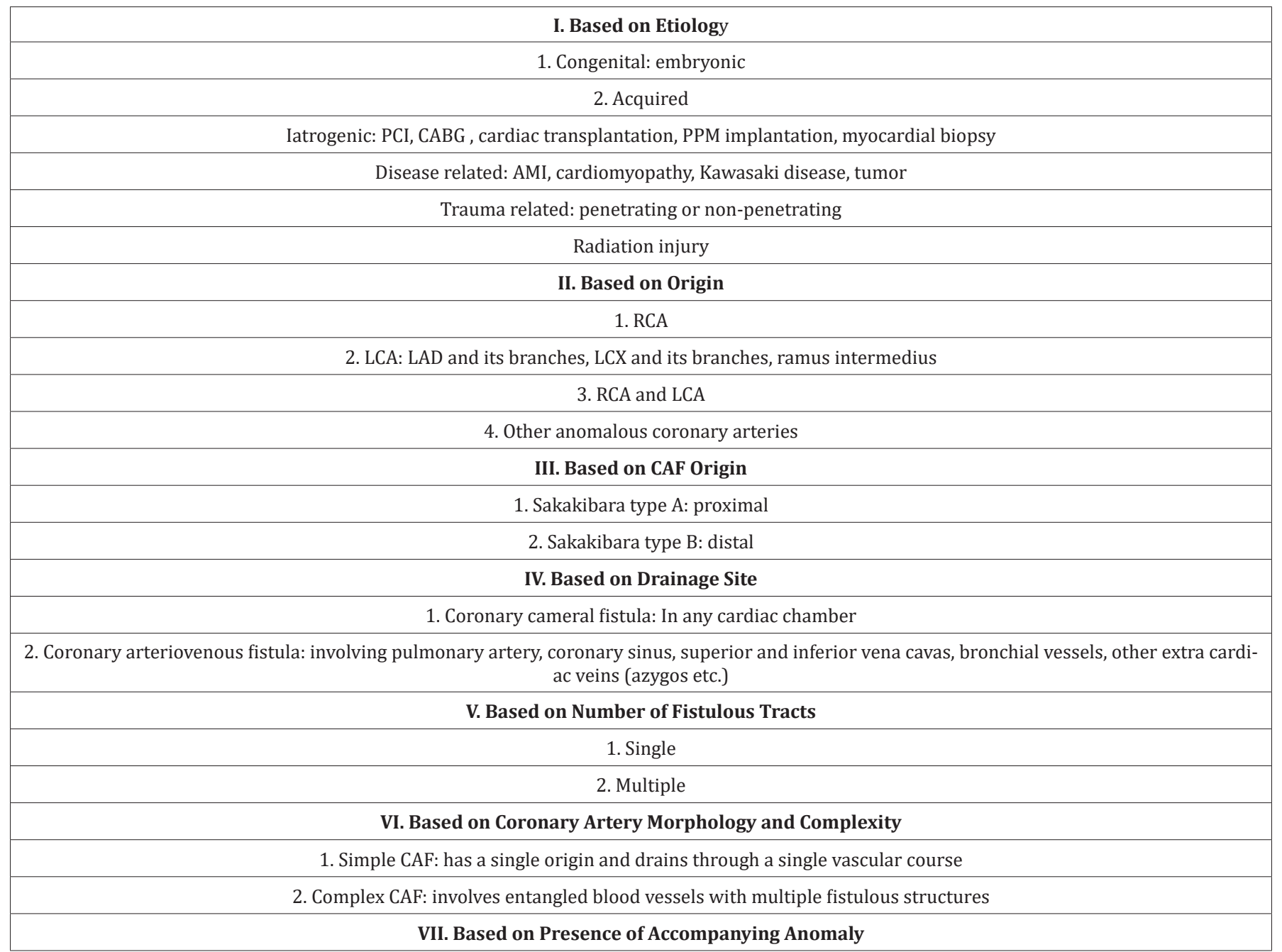


1. Isolated CAF: no accompanying anomaly

2. CAF accompanied by ventricular septal defect, patent ductus arteriosus, Tetralogy of Fallot etc.

Small coronary artery fistulae are usually asymptomatic and incidentally detected on coronary angiograms whereas large fistulae can lead to heart failure, MI, angina, arrhythmias, endocarditis, thrombosis/embolization, sudden cardiac death, premature atherosclerosis or rupture leading to Pericardial effusion.

Specialized diagnostic procedures are employed for the detection of these fistulae. Of these, color Doppler echocardiography is most easily available. CT angiography is especially helpful and can delineate the anatomy at times better than invasive angiography (particularly 3D multiplanar imaging with volume rendering gives excellent anatomical details). Magnetic resonance imaging can also be employed but gives less information about fistulous origin and drainage site).

Coronary artery fistulae, if small, need observation only but, if large or symptomatic, should be closed. This can be done either percutaneously or surgically. Percutaneous options for closure are coils, detachable balloons, umbrella devices, vascular plugs, covered stents and ductal occluders. Our patient had a small coronary cameral fistula originating from proximal Right
Coronary artery and draining into Right Atrium, Figure 2B (yellow arrow). Being small, no intervention is needed for it at present and it is highly unlikely to cause myocardial ischemia by steal phenomenon.

\section{Ventricular fibrillation}

Ventricular fibrillation, a complication of sinister proportions, occurs infrequently during coronary angiography with modern equipment and expertise. Its incidence in various large studies has been reported from 0.05 to $0.4 \%$. [8,9]. However, because of its ominous and lethal consequences, an in-depth knowledge of its occurrence is mandatory for all the operators. R West et al. [10] in their confidential audit of 211,645 cardiac catheterization procedures done in a decade (1990-1999) across England and Wales, found the complication rates to be low but neither negligible nor irreducible [10]. There were 1614 (0.76\%) complications with $164(0.077 \%)$ procedure-related deaths. Of the complications noted, 553 subjects $(0.25 \%)$ had ventricular arrhythmias, constituting the major segment. The main causes of ventricular fibrillation during coronary angiography are listed in Table 2 .

Table 2: Causes of ventricular fibrillation during coronary angiography.

\begin{tabular}{|c|r|}
\hline 1. Ischemia & Coronary artery stenosis (especially in 3VD and post CABG cases), spasm, dissection and ectasia. \\
\hline 2. Inappropriate Manipulation & Catheter occlusion/impaction, deep intubation, super-selection or myocardial stimulation. \\
\hline 3. Contrast Material & High osmolar ionic agents, long or rapid injections and contrast toxicity. \\
\hline 4. Hypokalemia & Prolonged duration or increased dispersion. \\
\hline 5. Related to QTc & \\
\hline 6. Unclear & \\
\hline
\end{tabular}

Ishemia can cause VF due to multiple reasons. The coronary fistula in the subject under discussion is small and no intrinsic vascular abnormality causing ischemia and subsequent arrhythmia has been noted. Thus, ischemia can be ruled out as a cause of ventricular fibrillation in this case.

Contrast material can induce VF by virtue of its osmolality or ionic nature. A marked reduction in the incidence of VF has been noted by the use of non-ionic agents of low osmolality. Joseph Misri et al. [11] in their study of two cohorts of 2000 subjects each undergoing coronary angiography by osmolar ionic contrast agent (group I) and low osmolar non-ionic contrast (group II), found that incidence in the second group was remarkably low $(1 \%$ vs. $0.4 \%$; $<<0.03$ ] [11]. Calcium sequestration by agents present in angiographic dye lowers serum Calcium level, which can precipitate ventricular arrhythmias. David K Murdock et al compared two dyes; the first group (A-2500 patients) was given dye containing disodium calcium EDTA (non-calcium sequestering) while the second group (B-2000 patients) had coronary angiography with dye containing EDTA and sodium citrate (calcium sequestering) [12]. The incidence of ventricular fibrillation was significantly less in group A as compared to group B $(0.1 \%$ vs $0.6 \%$; p < $<.02)$.The dye used, being of low osmolality and non-ionic, is less likely to act as a precipitant of $\mathrm{VF}$ in this patient.

Hypokalemia (defined as serum $\mathrm{K}<3.5 \mathrm{mEq} / \mathrm{L}$ ) is a known cause of VF by virtue of various mechanisms like prolonged ventricular repolarization (due to inhibition of outward K current with increased propensity of after-depolarizations), slowed conduction and abnormal pacemaker activity [13]. Changes in extracellular $\mathrm{K}$ alter membrane equilibrium of Potassium [Ek] as per Nernst Equation which causes more membrane hyperpolarization and increased $\mathrm{I}_{\mathrm{f}}$ (funny channel) activity and steeper slope of phase 4, resulting in enhanced ectopic pacemaker activity. 
Serum K must be checked for every patient undergoing coronary angiography and, if found low, should be appropriately corrected. This could not be the cause of arrhythmia in this patient as her serum Potassium level was $4.0 \mathrm{mEq} / \mathrm{l}$, which is well within the normal range.

\section{QT interval}

QT interval estimation receives very little attention during routine cases of coronary angiography. It spans from the onset of ventricular depolarization (i.e. beginning of $Q$ wave) till the completion of repolarization (end of $\mathrm{T}$ wave). Its prolongation can result in increased propensity for ventricular arrhythmias, especially Torsades de Pointes (first demonstrated by Desertennes in 1966) and ventricular fibrillation. Yiyi Zhang et al. [14] in their remarkable analysis of 7828 participants of NHANES III study (age> 40 years), found a U-shaped relationship between QTc interval (analyzed by Linear regression formula) and total mortality, Figure 3 [14]. They further correlated QTc interval with mortality due to CVD and non-CVD and obtained the same results. This shows that mortality increases as normal range of QTc is crossed on either side.

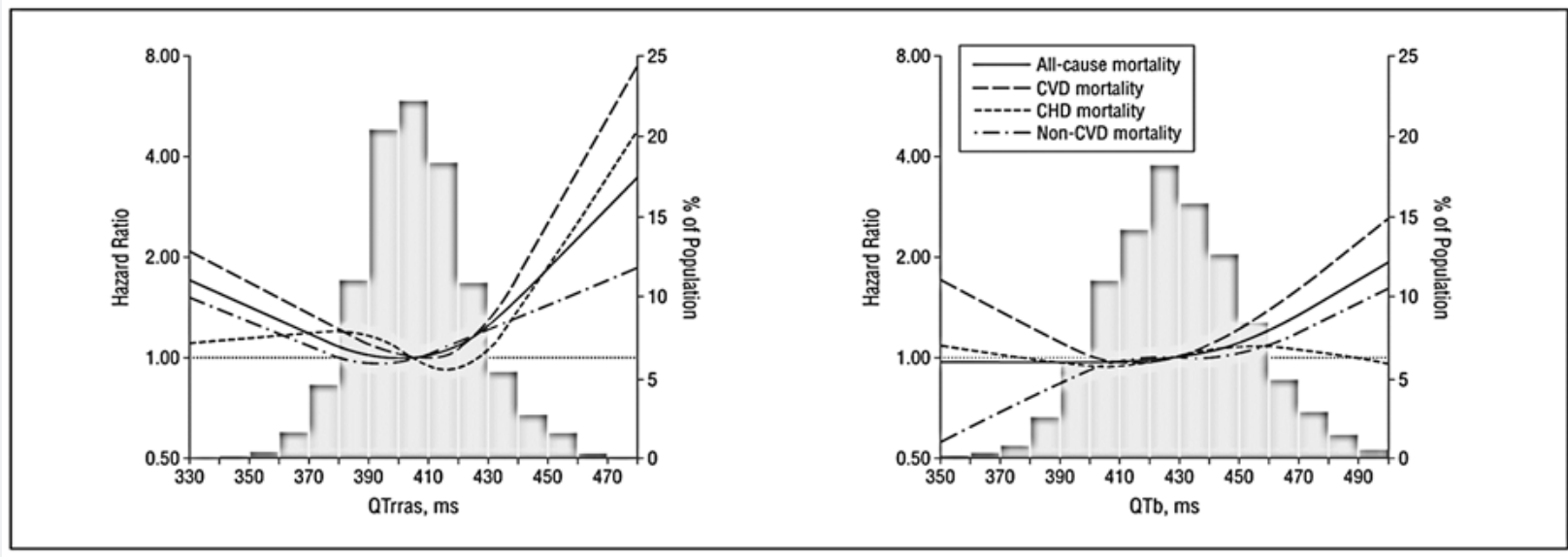

Figure 3: Multivariate-adjusted hazard ratios for total mortality rate and mortality due to CVD, CHD non-CVD.

For measurement of QT interval lead II is best, with lead I and V5 as alternatives. It should not be measured in leads III, aVL and V1. The beginning of $Q$ wave is usually clearly visible but the end of $\mathrm{T}$ wave may not be clearly demarcated as it trails down the baseline. Two methods have been proposed to mark the end of $\mathrm{T}$ wave, 'Tangent' and 'Threshold'.

Duration of QT interval is affected by heart rate being less at higher heart rates, hence, to rationalize this depreciation, QT interval is extrapolated to a HR of 60 beats per minute by various formulae, , and the value so obtained is known as Corrected QT interval, i.e. QTc.

Guidelines from ACC/AHA/HRS recommend QTc to be derived from Linear regression formula $(\mathrm{QTC}=\mathrm{QT}+0.154(1-\mathrm{RR})$. The maximum normal of QTc for males is $450 \mathrm{msec}$. and for females it is $470 \mathrm{msec}$. The patient under discussion had a QTc by this formula of $492 \mathrm{msec}$. which is clearly increased.

QT dispersion is the difference between the longest and shortest QT interval in the 12 leads of one ECG. This is also an indicator of mortality risk, even more so than QTc (RR > 1.26). Normally it does not exceed $80 \mathrm{msec}$.
Prolonged QT interval is a favorable substrate for the initiation of VF, particularly during coronary angiography as various other factors like ischemia, manipulation and contrast material may prolong it further, precipitating VF easily. J A Arrowood et al. [15], in a retrospective study of patients who had VF $(\mathrm{N}=26)$ during coronary angiography, found that QTc interval was significantly more in these patients than normal $(\mathrm{QTc}=0.4 \pm 0.04$ vs $0.42 \pm$ 0.03; $\mathrm{p}<0.001$ ).

Cardiac myocyte action potential is dependent upon the activity of ionic channels which control the movement of four principal ions, i.e. Sodium (Na), Potassium (K), Calcium (Ca) and Chloride $(\mathrm{Cl})$. They have distinct channels. Ion channels are either voltage-gated or ligand-gated. These channels are actually proteins coded by specific genes.

The activity of these ion channels with the direction of movement of ions across them and the corresponding phase of action potential are shown in Figure 4, [16].

Sodium channel is of one type only and opens during phase 0 of action potential when the resting membrane potential reaches a threshold. Its opening and closing is almost instantaneous (in $2 \mathrm{msec}$ ). 


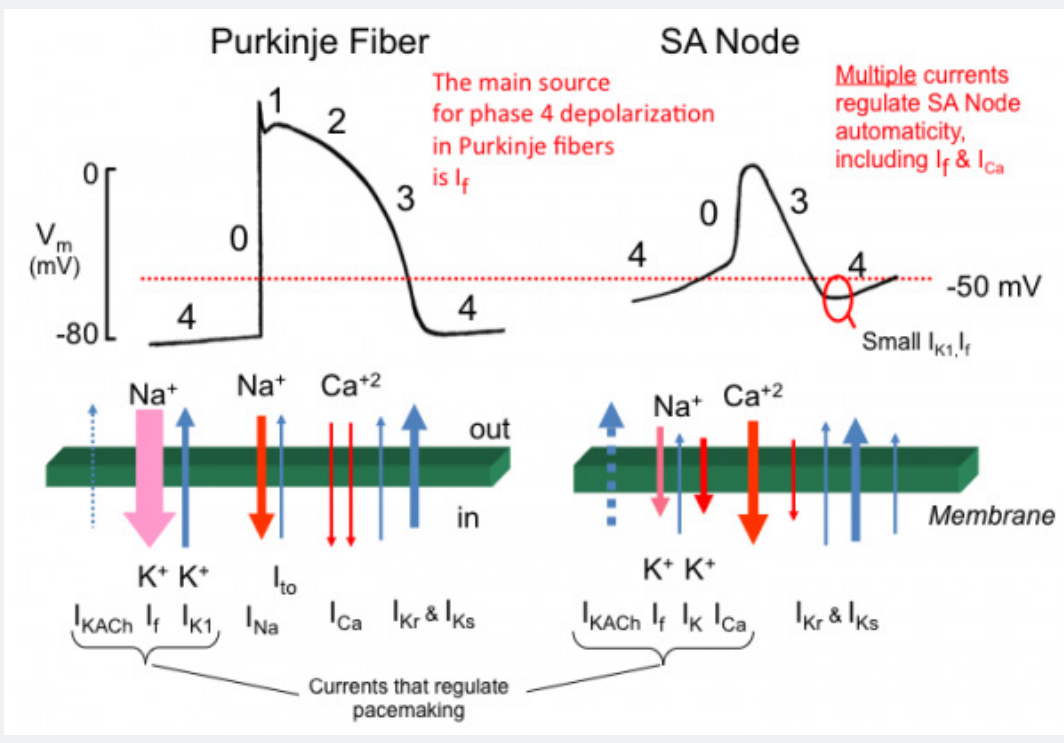

Figure 4: Action potential curves of purkinje fibres and SA node with corresponding ions involved.

The potassium channels are of three types:

a) $\mathrm{IK}_{\text {to1 }}$ (transient outward) which opens during phase 1 of action potential and causes outward movement of Potassium ions producing a notch in AP curve.

b) Second type of channels are known as delayed rectifier. These are of three types, ultra- rapid $\left(\mathrm{IK}_{\mathrm{urp}}\right)$ rapid $\left(\mathrm{IK}_{\mathrm{r}}\right)$ or slow $\left(\mathrm{IK}_{\mathrm{s}}\right)$. These open during phase 2 and 3 of AP curve and cause outward movement of potassium ions.

c) Third type is known as inward rectifier channel which opens during repolarization phase (phase 3) and resting membrane potential (phase 4) and can cause movement of Potassium ions on either side of the membrane.

There are two types of voltage-gated Calcium channels, L-type (long lasting) and T-type (transient). L-type is important with regard to cardiac AP. It opens during phase 2 causing inward movement of Calcium ions. Calcium ions activate the Ryanodine receptor on sarcoplasmic reticulum causing more release of Calcium in cytosol, affecting myocyte contraction. Because of these ions there is a prolonged phase 2 (plateau) in the action potential of cardiac myocyte.
Chloride channels, $\mathrm{I}_{\text {to2, }}$ are activated by Calcium ions during phase 2 and help maintain the plateau of phase 2 .

Besides these, there are funny $\left(\mathrm{I}_{\mathrm{f}}\right)$ channels, also known as HCN (hyperpolarization activated, cyclic nucleotide gated). These are activated by hyperpolarization of membrane and both sodium and potassium ions move across them (which is why they are called funny channel) to increase the RMP to its threshold of discharge during phase 4 . During phase 4 , sodium ion activated Na-K pump moves Na ions outwards and K ions inwards, whereas sodium calcium exchanger (NCX), activated by Calcium ions, moves Calcium ions outward and Na ions inward.

The prolongation in QT interval results from defective ionic channels most commonly the delayed Potassium rectifier (IKr and IKs) which results either in rapid Na ions inward movement or delayed $\mathrm{K}$ ion outward movement, either of which could result in prolongation of the repolarization phase of action potential.

A prolonged QT interval could either be congenital or acquired. Lia Crotti et al. [17] have summarized the types of Congenital long QT syndrome affecting 1 in 2500 persons, with genes involved, as shown in Table 3 [17].

Table 3: Types of congenital long QT syndromes, gene association and prevalence.

\begin{tabular}{|c|c|c|}
\hline LQTS TYPE & GENE & Prevalence (\% of all Genotyped Cases) \\
\hline LQT1 and JLN1 (AR) & KCNQ1 & 350 \\
\hline LQT2 & KCNH2 & $10-15$ \\
\hline LQT3 & SCNA5 & \\
\hline LQT4 & ANK2 \\
\hline
\end{tabular}




\begin{tabular}{|c|c|c|}
\hline LQT5 (RWS) and JLN2 & KCNE1 & \\
\hline LQT6 & KCNE2 & \\
\hline LQT7 (Anderson-Twail syndrome) & KCNJ2 & \\
\hline LQT8 (Timothy syndrome) & CACNA1c & \\
\hline LQT9 & CAV3 & \\
\hline LQT10 & SCN4B & \\
\hline LQT11 & AKAP9 & SNTA1 \\
\hline LQT12 & KCNJ5 & \\
\hline LQT13 &
\end{tabular}

It is not necessary that a person with prolonged QT interval will have ventricular arrhythmias. Certain factors act to precipitate this catastrophe and this has aptly been called a "gene-environment interaction". The factors which can precipitate ventricular arrhythmias in a person with prolonged QT interval have been summarized in Table 4 .

Table 4: Precipitating Factors for Ventricular Arrhythmias In Subjects With Prolonged QT Interval.

\begin{tabular}{|c|c|c|c|c|c|}
\hline Age & \multicolumn{5}{|c|}{ Elderly } \\
\hline Sex & \multicolumn{5}{|c|}{ Females } \\
\hline LV Morphology & \multicolumn{5}{|c|}{ LVH } \\
\hline Electrolytes & \multicolumn{5}{|c|}{ Hypokalemia, Hypocalcemia, Hypomagnesemia } \\
\hline Endocrine & \multicolumn{5}{|c|}{ Hypothyroidism, Diabetes Mellitus (Type II), Addison's disease. } \\
\hline Drugs & $\begin{array}{l}\text { Anitarrhythmics } \\
\text { Amiodarone Sotalol } \\
\text { Quinidine Procain- } \\
\text { amide Dofetilide } \\
\text { Ibutilide }\end{array}$ & $\begin{array}{l}\text { Antimicrobials Levofloxacin Cip- } \\
\text { rofloxacin Gatifloxacin Moxiflox- } \\
\text { acin Clarithromycin Erythromy- } \\
\text { cin Ketoconazole Itraconazole }\end{array}$ & $\begin{array}{l}\text { Antidepressants Ami- } \\
\text { tryptiline Desipramine } \\
\text { Imipramine Doxepin } \\
\text { Flouxetine Sertraline } \\
\quad \text { Venlaflaxine }\end{array}$ & $\begin{array}{l}\text { Antipsychotics } \\
\text { Haloperidol Dro- } \\
\text { peridol Quetiapen } \\
\text { Thioridazine } \\
\text { Ziprasidone }\end{array}$ & $\begin{array}{l}\text { Others Cisapride } \\
\text { Sumatriptan } \\
\text { Zolmitriptan Ar- } \\
\text { senic Dolasetron } \\
\text { Methadone }\end{array}$ \\
\hline Arthritis & \multicolumn{5}{|c|}{ Rheumatoid } \\
\hline
\end{tabular}

Certain findings during angiography can point to the cause of ventricular fibrillation. Ischemia as a cause produces marked ST segment depressions or elevations prior to its onset. If provocated by contrast material bradycardia, prolongation of PR or QT interval, QRS axis rotation or alteration in T wave amplitude may be noted. No such changes in ECG were noted in this patient and the arrhythmia occurred all of a sudden.

No such case has been reported before, the closest that comes to it is one mentioned by Abdulselam Ilter et al. [18] when a patient with Coronary artery fistula was being taken to catheterization laboratory and the patient went into ventricular fibrillation just before the procedure [18]. VF did not occur during the procedure and in this case the third problem, prolonged QT interval, had not been reported. Hence, this, to my knowledge, is the first reported case with these three entities.

\section{Conclusion}

Ventricular fibrillation occurring during routine coronary angiography is a rare event, but can happen with prolonged QT interval. Although Coronary artery fistula is another rare entity reported to cause VF, but only in multiple, large and tortuous cases. This patient had it most likely because of prolonged QT interval as the size of CAF was small. However, the simultaneous accumulation of three rare entities in a single patient are worth reporting and emphasize the importance of QTc estimation beforehand in every case so that the attending personnel will be extra vigilant to deal any arrhythmic emergency.

\section{Consent and Approval}

Written informed consent was taken from the patient for publication of this case with medical record. Approval was also taken from hospital administrator in this regard.

\section{Acknowledgment}

Thanks are due to Ms. Huffsa Imran for typing and proof reading the manuscript.

\section{References}

1. Al-Khatib SM, LaPointe NM, Kramer JM, Califf RM (2003) What Clinicians should Know about the QT interval. JAMA 289(16): 21202127

2. Qureshi SA (2006) Coronary arterial fistulas. Orphanet Journal of Rare Diseases 1(51): 1-6. 
3. Hancock EW, Deal BJ, Mirvis DM, Okin P, Kligfield P, et al. (2009) AHA/ACCF/HRS Recommendations for the Standardization and Interpretation of the Electrocardiogram Part IV and V: Electrocardiogram Changes Associated With Cardiac Chamber Hypertrophy A Scientific Statement From the American Heart Association Electrocardiography and Arrhythmias Committee, Council on Clinical Cardiology; the American College of Cardiology Foundation; and the Heart Rhythm Society Endorsed by the International Society for Computerized Electrocardiology. J Am Coll Cardiol 53(11): 9821002.

4. Chiu CZ, Shyu KG, Cheng JJ, Lin SC, Lee SH, et al. (2008) Angiographic and clinical manifestations of coronary fistulas in Chinese people. Circ J 72(8): 1242-1248

5. Vavuranakis M, Bush CA, Boudoulas H (1995) Coronary artery fistulas in adults: incidence, angiographic characteristics, natural history. Catheterization and Cardiovascular Diagnosis 35(2): 1-2.

6. Levin DC, Fellows KE, Abrams HI (1978) Hemodynamically significant primary anomalies of the coronary arteries. Angiographic aspects. Circulation 58(1): 25-34.

7. Yun G, Nam TH, Chun EJ (2018) Coronary artery fistulas: pathophysiology, imaging findings, and management. Radiographics 38(3): 688-703.

8. Johnson LW, Lozner EC, Johnson S, Krone R, Pichard AD, et al. (1989) Coronary Arteriography 1984-1 987: A Report of the Registry of the Society for Cardiac Angiography and Interventions. I. Results and Complications. Catheterization and Cardiovascular Diagnosis 17(1): 5-10.

9. Chen J, Gao L, Yao M, Chen J (2008) Ventricular arrhythmia onset during diagnostic coronary angiography with a $5 \mathrm{~F}$ or $4 \mathrm{~F}$ universal catheter. Rev Esp Cardiol 61(10): 1092-1095.
10. West R, Ellis G, Brooks N (2006) Complications of diagnostic cardiac catheterization: results from a confidential inquiry into cardiac catheter complications. Heart 92(6): 810-814.

11. Missri J, Jeresaty RM (1990) Ventricular fibrillation during coronary angiography: reduced incidence with nonionic contrast media. Catheterization and Cardiovascular Diagnosis 19(1): 4-7.

12. Murdock DK, Johnson SA, Loeb HS, Scanlon PJ (1985) Ventricular fibrillation during coronary angiography: Reduced incidence in man with contrast media lacking calcium binding additives. Catheterization and Cardiovascular Diagnosis 11(2): 853-859.

13. Osadchii OE (2010) Mechanism of hypokalemia-induced ventricular arrhythmogenicity. Fundamentals \& Clinical Pharmacology 24(5): 547-559.

14.Zhang Y, Post WS, Dalal D, Blasco-Colmenares E, Tomaselli GF, et al (2011) QT interval duration and mortality rate. Arch Internal Med 171(19): 1727-1733.

15. Arrowood JA, Mullan DF, Kline RA, Engel TR, Kowey PR (1987) Ventricular fibrillation during Coronary angiography: The precatheterization QT interval. Journal of Electrorocardiology 20(3): 255-259.

16.Introduction_to_cardiac_physiology_electrophysiology_TUSOM/ PHARMWIKI.

17. Crotti L, Celano G, Dagradi F, Schwartz PJ (2008) Congenital long QT syndrome. Orphanet J Rare Dis 3: 18-33.

18. İlter A, Tusun E, Cirit A (2018) Multivessel coronary-cameral fistulas associated with ventricular fibrillation: an unusual case. Turk kardiyol Dern Ars 46(5): 392-395.

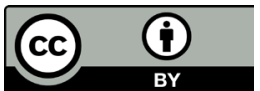

This work is licensed under Creative Commons Attribution 4.0 License DOI: 10.19080/JOJCS.2020.11.555805 